\title{
Trap seeker and digger: the dual identity of the solvated electron in methanol
}

\author{
Jinggang Lan, ${ }^{*} \dagger$ Yo-ichi Yamamoto, ${ }^{\ddagger}$ Toshinori Suzuki, ${ }^{\ddagger}$ and Vladimir V. \\ Rybkin*, \\ $\dagger$ Department of Chemistry, University of Zurich, Winterthurerstrasse 190, Zurich 8057, \\ Switzerland \\ $\ddagger$ Graduate School of Science, Kyoto University, Kyoto, Japan \\ E-mail: jinggang.lan@chem.uzh.ch; vladimir.rybkin@chem.uzh.ch
}

\begin{abstract}
The structure of the solvated electron in methanol is less studied but more complicated than the one of the hydrated electron. In this condensed-phase first principles molecular dynamics study we reveal the nature of the recently discovered shallow and deep trap states of the excess electron and suggest a more complex picture including four bound cavity states classified by the number of the hydroxy-groups coordinated to the electron, their binding energy gradually increasing with the OH-coordination. The initial shallow bound states are formed via a transient diffusion mechanism, in a trap-seeking fashion, whereas, deeper bound states are formed via a slower methanol molecules reorientation. Despite apparent similarity of the absorption spectrum of the solvated electron in methanol to that in water, the origin of the absorption maximum is drastically different. The previously assumed model of hydrogenic transitions ( $\mathrm{s}-\mathrm{p}$ etc.) as is the case in water does not hold for methanol. Instead, the main bands arise due to the charge-transfer states, promoting the excess electron to the nearby cavity,
\end{abstract}


naturally abundant in this solvent. We propose an alternative simple model to describe electronic states of the solvated electron in methanol: the double square well.

\section{Introduction}

Since their discovery of solvated electrons in $1960 \mathrm{~s},{ }^{1} e_{\text {sol }}^{-}$, have attracted attention as prototype radicals and reducing agents. ${ }^{2}$ Due to their non-typical chemical nature (no classical formula can be assigned for $e_{\text {sol }}^{-}$), transient character and low concentrations of solvated electron have avoided direct structure observation by such methods as diffraction and nuclear magnetic resonance: the structural information is indirectly extracted from electronic, vibrational and electron paramagnetic resonance spectroscopy data. This opens wide possibilities for theoretical modelling, which is, however, as challenging as experimental structure structure determination.

Most attention has been attracted to the electrons solvated in water and ammonia. ${ }^{3-5}$ The latter one is remarkably stable and belongs to textbook chemistry (the so called "blue solutions") and has found industrial application for the Birch reduction. ${ }^{5}$ The aqueous electron, $e_{a q}^{-}$, is in focus due to the abundance of water and relevance for radioactive DNA damage. After an intense debate on whether a hydrated electron occupies a cavity or not the non-cavity hypothesis has been practically ruled out due to recent theoretical and experimental advances. ${ }^{2}$ The aqueous excess electron occupies a 4 to 5 coordinated cavity with $\mathrm{O}-\mathrm{H}$ moieties of water molecules pointing towards its center. The spin density resides to a large extent within the cavity, ca. $20 \%$ of it spreading beyond the cavity, negative spindensity regions being present. ${ }^{2} e_{a q}^{-}$is formed within $0.5 \mathrm{ps}$ localizing via a very short-lived transient state, known as wet electron. ${ }^{6}$

The dynamic nature of the excess electron solvated in methanol, $e_{m e t}^{-}$, is less studied, but more interesting and challenging than its counterparts in simpler liquids. Indeed, time-

resolved experiments have revealed two trapped states of $e_{m e t}^{-},{ }^{7}$ not known for water and 
ammonia: the shallow and the deep trap states, both within the ground electronic state. The shallow trap state was first observed in low-temperature (4-77 K) glasses. ${ }^{8,9}$ At temperatures above $77 \mathrm{~K}$, the deep trap state was identified and assigned to a cavity formed by thermal motion activated at higher temperatures. Very recent femtosecond pump-probe experiments on $e_{m e t}^{-}$at ambient conditions revealed both shallow and deep trap states present for ca. 150 ps, after that the former completely converting into the latter. The two states have distinctly different binding energies: $2.1 \mathrm{eV}$ (shallow) and $3.4 \mathrm{eV}$ (deep).

On the theory side, only few theoretical works on the solvated electron methanol have been published, mainly dealing with clusters. Based on cluster calculations in a dielectric medium, Kumar et al. ${ }^{10}$ suggested a bulk structure, similar to that of $e_{a q}^{-}$: a tetrahedral cavity formed by four OH-groups. According to the computed electron binding energy, it likely corresponds to the deep trap state. Similar structures are observed in the condensed-phase molecular dynamics simulations using simple electronic-structure models. ${ }^{11-13}$ Some insights from the cluster and bulk simulations ${ }^{11,14,15}$ suggest that methyl groups also participate in binding the excess electrons (especially in pre-equilibrium structures in clusters), hinting at a more complicated picture. At the same time, there is no theoretical evidence for the shallow trap state. Identifying the nature of both states and their conversion mechanism in the bulk is an intriguing and challenging task for theory.

As soon as excited electronic states of the solvated electron in methanol are considered, the UV-visible absorption spectrum of $e_{m e t}^{-}$differs significantly from that of $e_{a q}^{-} \cdot{ }^{16-18}$ Although both are broad peaks with closely lying maxima (1.7 eV for water and $2.0 \mathrm{eV}$ for methanol), the spectrum of $e_{\text {met }}^{-}$exhibits significant absorption beyond $3.0 \mathrm{eV}$. The absorption maximum of $e_{a q}^{-}$corresponds to the transitions from the s-like spin-orbital to the three p-like virtual spin-orbitals, whereas higher-energy hydrogenic and continuum states form the long "blue tail". ${ }^{19}$ Based on the one-electron Hamiltonian model, the absorption maximum of $e_{m e t}^{-}$was found to be of the same nature as in $e_{a q}^{-}{ }^{11,20}$ This model was also assumed in interpreting experimental pump-probe spectra. ${ }^{21}$ At the same time, absorption beyond $3.0 \mathrm{eV}$ has not 
been explained and it is unlikely that the hydrogenic one-particle picture can account for it. Moreover, lifetimes of excited states and bleaching of solvated electrons in alcohols are significantly different from those in water and correlate with the carbon chain length ${ }^{18,21,22}$ suggesting a different qualitative picture.

Here we disentangle the complex nature of the ground state of the solvated electron in methanol by condensed-phase molecular dynamics (MD) simulations based on hybrid density functional theory (DFT) and second-order Møller-Plesset perturbation theory (MP2) and followed by time-dependent DFT calculations of electronic spectra, suggesting a new interpretation of experimental observations.

\section{Computational methods}

\section{Molecular dynamics}

We performed two main sets of production MD simulations for different system sizes: 39 and 55 methanol molecules and an excess electron, 5 trajectories each. We traced the timeevolution of the system after the addition of the excess electron to the neat liquid methanol at ambient conditions in an NVE ensemble. Each production trajectory has been integrated up to 5 ps. Initial conditions have been taken from the NVT trajectories of liquid methanol equilibrated at $300 \mathrm{~K}$ and corresponding to the experimental density.

Simulations have been performed using hybrid DFT functional $\operatorname{PBE}(\alpha)$ with $50 \%$ of exact exchange with non-local van der Waals correction ${ }^{23}$ using a triple-zeta quality basis set GTHTZV2P ${ }^{24}$ proven to provide spin densities and dynamics similar to those of MP2. ${ }^{4,25}$

For the smaller system (39 molecules), we have reintegrated two shorter trajectories (ca. 1 ps each) at the MP2 level of theory ${ }^{26,27}$ with triple-zeta quality basis sets $^{28}$ to check the main dynamic features at a higher level of electronic structure theory, which is significantly more computationally demanding. ${ }^{29}$

All calculations have been performed using CP2K package ${ }^{30}$ based on Goedecker-Teter- 
Hutter $(\mathrm{GTH}) \cdot{ }^{31}$ Details of the electronic structure set-up are given in the Supplementary Information.

\section{Binding energies}

Band alignment under periodic boundary conditions is a non-trivial task. ${ }^{32,33}$ Here we base our calculations of vertical binding energies (VBE) on two assumptions. First, semi-core 2s-levels of oxygen are not altered by excess electron addition. Second, the excess electron immediately after injection is not bound, which corresponds to the experimental observations. ${ }^{7}$ Consequently, VBE for the $i$ th configuration is calculated as follows:

$$
\mathrm{VBE}_{i}=E(\mathrm{SOMO})_{i}-E\left(\mathrm{O}_{2 s}\right)_{i}-\left(E(\mathrm{SOMO})_{0}-E\left(\mathrm{O}_{2 s}\right)_{0}\right),
$$

where $E(\mathrm{SOMO})$ is the energy of the singly-occupied molecular orbital, corresponding to

the excess electron, $E\left(O_{2 s}\right)$ is the energy of the oxygen $2 \mathrm{~s}$ band and 0 index corresponds to the first frame. The $\operatorname{PBE}(\alpha)$ with $50 \%$ of exact exchange functional was used to compute one-particle energy levels for frames extracted from both DFT and MP2 trajectories.

\section{Electronic spectra}

Electronic spectra have been computed by time-dependent density functional perturbation theory (DFPT) using $\operatorname{PBE}(\alpha)$ with $50 \%$ of exact exchange with non-local with triple-zeta quality basis set GTH-TZV2P. ${ }^{24}$ We have computed 20 excited states for each of the frames selected from the MD trajectories of the larger system. 


\section{Results}

\section{Structure and dynamics}

The structure of the solvated electron in methanol is more complex than in simpler liquid,

e.g. water $^{3,4}$ or ammonia. ${ }^{5}$ Types of cavities occupied by the excess electron in methanol are defined by the bipolar nature of the solvent, consisting of two groups: bulky non-polar methyl- and a small polar hydroxy-group. Thus, we separate the following cavity types (see Figure 1 and Figure 2(a-c)):

1. $\mathrm{CH}_{3}$-cavity: formed by 4-6 methyl groups;

2. 1OH-cavity: formed by one hydroxy- and several methyl-groups;

3. 2OH-cavity: formed by two hydroxy- and two methyl-groups;

4. 3 OH-cavity: formed by three hydroxy- and one methyl-group;

5. $4 \mathrm{OH}$-cavity: formed by four hydroxy-groups.

$\mathrm{CH}_{3}$-cavities occur naturally in liquid methanol. Therefore, in most simulations the excess electron is initially localized in one of them, often forming a compact and relatively isotropic spin-density distribution, although the electron is only weakly bound (structures with small gyration radius, anisotropy, and vertical binding energy (VBE) in Figure 2(e)). As soon as a hydrogen bond is broken in the vicinity, forming a polar trap, the dangling OH-bond, the electron flows to this newly opened cavity. The process is similar to transient diffusion (See Figure.1a-c) described recently for aqueous solvated electron ${ }^{3}$ and results in a $1 \mathrm{OH}-$ cavity formation, some distance away from the inital localization. The $\mathrm{CH}_{3}$-cavity stage can also be bypassed, so that the electron localizes in the $1 \mathrm{OH}$-cavity from the beginning if a broken hydrogen bond happens to exist at the time of electron's injection. Thus, at the early stages, solvated electron in methanol "looks for" and follows the most energetically favourable localization site available, which may be either $\mathrm{CH}_{3}$ - or $1 \mathrm{OH}$-cavity. This is consistent with 
the experimentally anticipated picture of the excess electron as "a trap-seeker and not a trap-digger" ${ }^{7-9}$ at the early stage.

The picture changes dramatically when $2 \mathrm{OH}-, 3 \mathrm{OH}$ - and $4 \mathrm{OH}$-cavities are being formed: the solvated electron now behaves as a "trap-digger". With time, the methanol molecules coordinated to the excess electron with their $\mathrm{CH}_{3}$-groups reorient themselves to coordinate with their OH-groups, whereas the electron "stays in its place". This may occur with one or two molecules reorienting at a time (as shown in Figure 1). The process is launched by the breaking of the donor hydrogen bond, formed by the hydroxyl of the molecule coordinating to the cavity with its methyl group. Then the methanol molecule rotates to coordinate the cavity with the now dangling $\mathrm{OH}$-group. This mechanism is similar to the event triggering the reaction of the aqueous electron with $\mathrm{CO}_{2},{ }^{25}$ highlighting the importance of the second solvation shell in understanding the dynamics of solvated electrons.

The whole process generally follows the scheme shown in the equation:

$$
\begin{aligned}
\mathrm{CH}_{3}-\text { cavity } & \rightarrow 1 \mathrm{OH}-\text { cavity } \rightarrow 2 \mathrm{OH}-\text { cavity } \\
& \rightarrow 3 \mathrm{OH}-\text { cavity } \rightarrow 4 \mathrm{OH}-\text { cavity }
\end{aligned}
$$

Some stages of the scheme can be omitted or show up for only several dozens of fs as transient intermediates. It is worth noting here, that there was an important difference between our simulations of the smaller (39 molecules) and the larger (55 molecules) systems. In the latter, none of the 5 trajectories leads to the $4 \mathrm{OH}$-cavity formation within 5 ps. To compute the properties of this cavity type it has been prepared "by hand" and equilibrated. To summarize, for the larger system, three trajectories reached the state of $3 \mathrm{OH}$-cavity, and two trajectories reached the stage of $2 \mathrm{OH}$-cavity within 5 ps. For the smaller system, three trajectories reached the $4 \mathrm{OH}$-cavity, one - $3 \mathrm{OH}$-cavity, and one - $2 \mathrm{OH}$-cavity. MP2 simulations of the smaller system resulted in two trajectories, one tracing the formation of the $2 \mathrm{OH}$-cavity from the $\mathrm{CH}_{3}$-cavity via the $1 \mathrm{OH}$-cavity intermediate. The second captured the 
$3 \mathrm{OH}-$ to $4 \mathrm{OH}$-cavity transition via the methanol molecule reorientation. Both trajectories are consistent with their hybrid DFT counterparts.
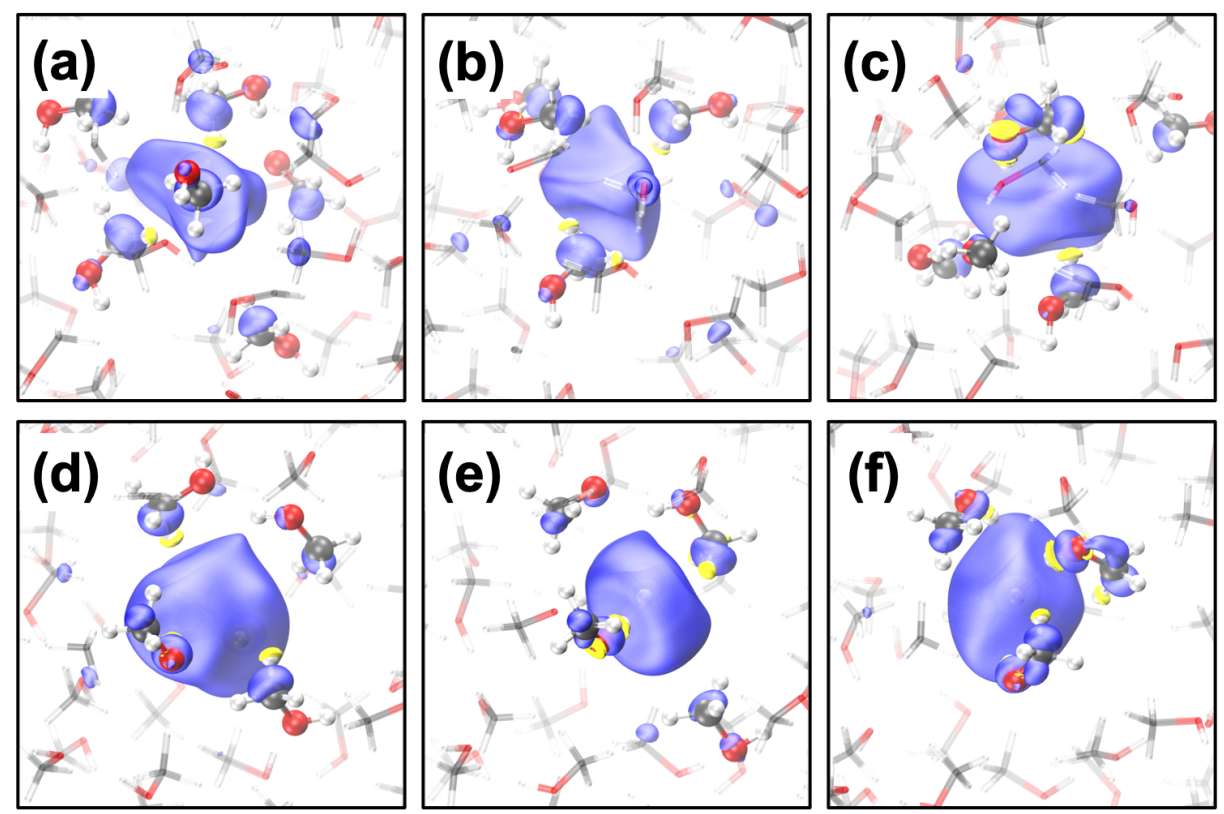

Figure 1: (a-c) Transient diffusion from the $\mathrm{CH}_{3}$-cavity to the $1 \mathrm{OH}$-cavity; (d-f) transition from the $2 \mathrm{OH}$-cavity to the $4 \mathrm{OH}$-cavity. Color code: oxygen (red), hydrogen (white) and positive spin density (blue), negative density (yellow) where the isovalue is 0.001 a.u.

A more detailed look at the structures is given by radial distribution functions (RDFs) of $\mathrm{C}, \mathrm{H}$, and $\mathrm{O}$ atoms with respect to the center of spin density distribution as shown in Figure 1(a-c). We generally observe, that with the increase in OH-coordination the structure becomes more ordered even in the case of $\mathrm{g}_{e-\mathrm{C}}$, where the first peak shifts gradually to the long-range region, becoming sharper. As for the $\mathrm{g}_{e-\mathrm{H}}$, the $\mathrm{CH}_{3}$ - and $1 \mathrm{OH}$-cavity have a very similar molecular pattern, while the RDF of $1 \mathrm{OH}$-cavity is more ordered as compared to the $\mathrm{CH}_{3}$ - one. Interestingly, the center of the spin-density distribution stays closer to the dangling $\mathrm{OH}$-groups in lower coordinated cavities, the corresponding peaks on $\mathrm{g}_{e-\mathrm{O}}$ and $\mathrm{g}_{e-\mathrm{H}}$ shifting to the long-range region.

As far the dynamics of structural change is considered, it is possible to deduce that since the conversion to the final state, $4 \mathrm{OH}$-cavity, is not achieved in all trajectories within 5 ps (in case of the larger system conversion is slower as no trajectory achieves this state) our 

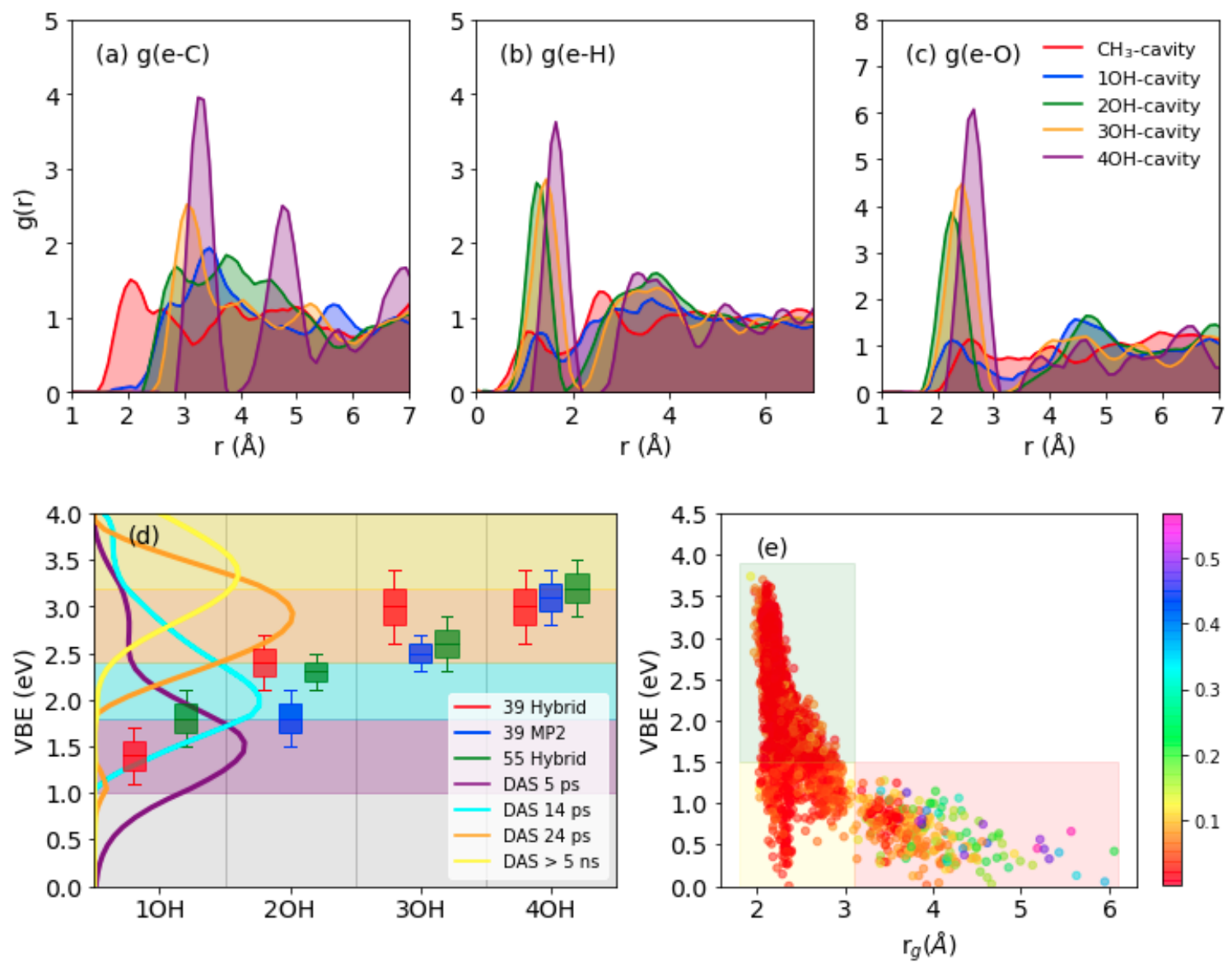

Figure 2: (a-c) Radial distribution functions for main structures (55 molecules): electron distribution center to carbon (a), hydrogen (b), oxygen (c). (d) Vertical electron binding energies (shifted by $0.4 \mathrm{eV}$ to compare with experimental results) of different cavity types as obtained from 39 molecule-system using hybrid functional (red), 39 molecules using MP2 frames (blue), and 55 molecules using hybrid functional frames (green). Decay associated spectra (DAS) calculated by global fitting of Photoemission spectra with four-step sequential kinetics model. The spectra are measured by liquid methanol excited by $9.3-\mathrm{eV}$ vacuum UV pulses and probed by 4.3-eV UV pulses (e)Vertical binding energies vs. spin density gyration radius (55 molecules). The color map corresponds to spin density distribution anisotropy. 
time-scales are in qualitative agreement with the experimental observations. Indeed, the full conversion to the final state happens at a much longer time-scale, ${ }^{7}$ over 100 ps. Nevertheless, better statistics is certainly needed for quantitative conclusions on transformation times.

\section{Binding energies}

Binding energies as obtained from our simulations for different structure types are shown in Figure 2, lower panels. We see that the VBE gradually increases with the OH-coordination. The numbers obtained from different trajectories generally agree with each other within error margins. When shifted ca. 0.4 above the energy range fits the energy range of the trap states almost exactly: between $1.5 \mathrm{eV}$ for the lower edge of the shallow-trap states and $3.4 \mathrm{eV}$ for the deep-trap one. ${ }^{7}$ Thus, although systematically underestimating the VBE, our calculations deliver accurate relative binding energies for the trap states. The picture, however, appears to be more complex than the thought of before: there are four rather than two bound states with broad overlapping VBE distributions.

Our theoretical findings have encouraged us to reinterpret the experimental results of Hara et al., who performed ultrafast photoemission spectroscopy study of liquid methanol, in which they excited methanol with $9.3 \mathrm{eV}$ vacuum UV pulses and interrogated the subsequent solvated electron formation using photoemission with UV pulses. ${ }^{7}$ It has been found that the

photoemission spectrum varies in the picosecond time scale and that the electron binding distributions (eBEs) can be categorized with shallow and deep trap states. These two trap states exhibited increasing eBE due to solvation with the time constants of 15 and 50 ps. Therefore, neither of these trap states was stationary. Alternatively, the spectral evolution of solvated electron could be expressed with chemical species with stationary spectra. In this case, the sequential formation of four species needed to be assumed. The decay times of these four species were determined to be $5.1,14,24 \mathrm{ps}$, and $>5 \mathrm{~ns}$, respectively. The eBE distributions, not presented by Hara et al., of these four species, are shown in Figure.2(d). The four species have average eBE values of $1.7,2.2,2.8$, and $3.3 \mathrm{eV}$, which are almost 
equally spaced. Thus, when one employs a model assuming a stationary eBE distribution for each chemical species, the analysis of the experimental results is consistent with our theoretical calculations. Close examination of Figure.2(d) reveals that the first species has a rather wide eBE distribution and its high energy tails exceed $3.0 \mathrm{eV}$. This implies that some of the electrons are directly trapped in the region where multiple $\mathrm{OH}$ bonds can stabilize the electron.

\section{Electronic spectra}

The nature of excited states and electronic spectra of the excess electron in methanol is dramatically different from this of the aqueous electron. In all OH-type (i.e. bound) cavities the main absorption peak corresponds to charge transfer, rather than to the s-type - p-type orbital transition within the cavity. The charge transfer takes place between the OH-cavity and one of the $\mathrm{CH}_{3}$-cavities, naturally occurring in liquid methanol as shown in Figure 3(c). $3 \mathrm{OH}-$ and $4 \mathrm{OH}$-cavity structures have the absorption maximum at ca. $2.2 \mathrm{eV}$, whereas the $2 \mathrm{OH}$-cavity structure has the absorption maximum at ca. $1.7 \mathrm{eV}$ (see Figure 3(c)). With a moderate blue shift of $0.2 \mathrm{eV}$, this corresponds to the experimentally observed absorption maxima of the shallow $(1.5 \mathrm{eV})$ and the deep trap $(2.0 \mathrm{eV})$ states. $1 \mathrm{OH}$-cavity structure absorbs less intensively and has a maximum at less than $1 \mathrm{eV}$. This makes it possible to complete the state assignment: $3 \mathrm{OH}$ - and $4 \mathrm{OH}$-structures correspond to the deep-trap state, whereas $1 \mathrm{OH}$ - and $2 \mathrm{OH}$-structures form the shallow-trap state. ${ }^{7}$

Moreover, we can describe the experimentally observed significant absorption beyond 3.0 $\mathrm{eV}$ and assign it to similar charge-transfer transitions. Intensive absorption in this region is neither observed for the hydrated electron (rather the "blue tail" ${ }^{19}$ ), nor predicted by the oneelectron Turi-Borgis (TB) Hamiltonian model in methanol. ${ }^{11}$ Conversely to our observation, the TB model assigns the maximum at $2.0 \mathrm{eV}$ to the three s-type - p-type orbital transitions. Very likely, it has to do with the nature of the model, which artificially confines the excess electron to a cavity, thus, preventing charge transfer upon excitation. 
To check this hypothesis, we have performed density functional embedded calculations on one of the $4 \mathrm{OH}$-cavity structures, using the four cavity-forming molecules as the embedded cluster. The embedding potential confines the electron to the cavity similar to the TB model. As a result of the embedded time-dependent density functional theory (TDDFT) calculation after removal of the spurious delocalized states ${ }^{19,32}$ we indeed observed the first three excitations to correspond to the s-type - p-type orbital transitions.
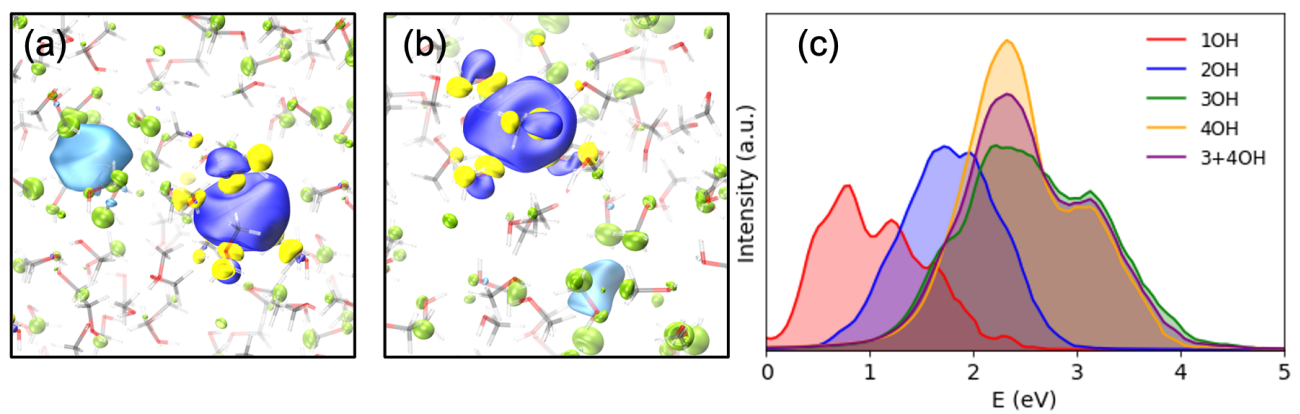

Figure 3: SOMO (blue - positive values, yellow - negative values) and the dominant virtual spin-orbital in the first excited state (light blue - positive values, green - negative values) for a typical (a) 2OH-cavity structure and (b) 4OH-cavity structure (shallow-trap ground state). Isovalue - 0.02 a.u; green, yellow - positive values; light blue and blue - negative values. (c) Simulated electronic spectra of the solvated electron in methanol, including 20 excited states.

The charge-transfer nature of the excited states of the solvated electron in methanol must have drastic effects on their dynamics and lifetimes. First, the internal conversion must be an important decay channel, with a mechanism similar to a transient diffusion from the $\mathrm{CH}_{3}$-cavity to the already existing $\mathrm{OH}$-cavity. Assuming a similar picture of the excess electron in the ethanol we can suggest that the lifetime of the excited state will then depend on the carbon chain length as it defines the spatial separation between the cavities: the longer the backbone, the larger the lifetime. This is exactly what is observed in the experiments. ${ }^{18,21,22}$ Thus, the charge transfer picture of the excited states explains the observations on the qualitative level. It is, on the other hand, hard to explain this behavior with the conventional s-p transition picture.

It is, however, possible to rationalize the charge transfer excitations via a simple one- 
dimensional double square-well model. We assign the well depths to those of the $\mathrm{nOH}-$ (ground) and $\mathrm{CH}_{3^{-}}$(excited) states. Their widths then correspond to the gyration radii, whereas the distance between the well is roughly equal to the molecule length. Solving the model for the ground and the first excited state using reasonable values for different state types one can obtain the energy difference close to the experimentally spectral maxima (see Table 1), whereas the wave functions are indeed mostly localized in the corresponding wells (see Figure 4). Moreover, if we increase the distance between the well corresponding to the ethanol molecule, we will get a consistent red shift as compared to methanol in agreement with experiment. ${ }^{16-18}$

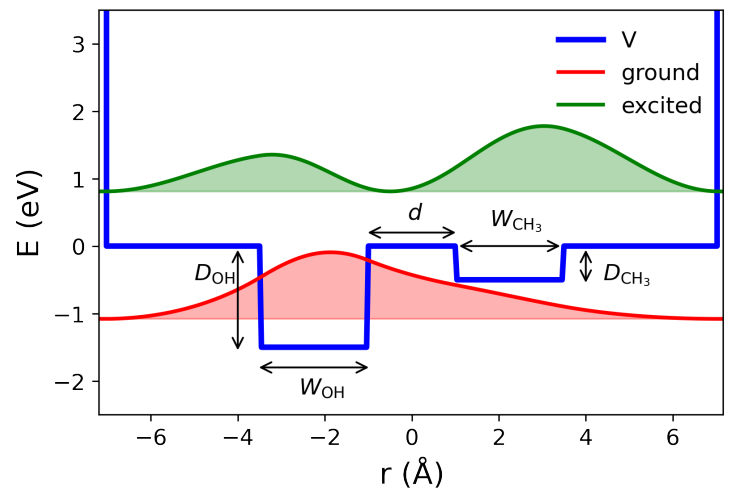

Figure 4: Double square well model: potential; energies and probability density distributions of the ground and first excited states. $W_{\mathrm{OH}}$ and $W_{\mathrm{CH}_{3}}$ are width of the two wells; $d$ is the distance between the wells; $D_{\mathrm{OH}}$ and $D_{\mathrm{CH}_{3}}$ are the well depths.

Table 1: Double square well model: parameters and results. $W_{\mathrm{OH}}$ and $W_{\mathrm{CH}_{3}}$ are width of the two wells, corresponding to the gyration radii of the states; $d$ is the distance between the wells, corresponding to the carbon backbone length; $D_{\mathrm{OH}}$ and $D_{\mathrm{CH}_{3}}$ are the well depths corresponding to the electron binding energies. $\Delta E$ is the energy gap between the ground and the first excited states. Notation is clarified in Figure 4

\begin{tabular}{l|c|c|c|c}
\hline & deep & shallow & shallow, edge & ethanol \\
\hline$W_{\mathrm{OH}}(\AA)$ & 2.2 & 2.5 & 2.5 & 2.2 \\
$W_{\mathrm{CH}_{3}}(\AA)$ & 2.5 & 2.5 & 2.5 & 2.5 \\
$d(\AA)$ & 2.0 & 2.0 & 2.0 & 3.0 \\
$D_{\mathrm{OH}}(\mathrm{eV})$ & 3.4 & 2.1 & 1.5 & 3.4 \\
$D_{\mathrm{CH}_{3}}(\mathrm{eV})$ & 0.5 & 0.5 & 0.5 & 0.5 \\
\hline$\Delta E(\mathrm{eV})$ & 1.9 & 1.5 & 1.3 & 1.8
\end{tabular}




\section{Conclusions}

In the first dynamic simulation of the condensed-phase excess electron in methanol based on high-level electronic structure theory, we trace the complex dynamics of the species. We identify the bound states of the solvated electron classified according to the number of $\mathrm{OH}-$ (and $\mathrm{CH}_{3^{-}}$) groups forming the confining cavity: from $1 \mathrm{OH}-$ to $4 \mathrm{OH}$-cavity. After electron injection, the electron typically occupies a naturally occurring cavity formed by $\mathrm{CH}_{3}$-groups and then flows to a more energetically favourable binding site - stochastically formed free OH-group - in a transient diffusion process. This trap-seeking behavior is then changed to a trap-digging one: cavity-forming methanol molecules reorient themselves to coordinate with $\mathrm{OH}$-group rather than with the $\mathrm{CH}_{3}$-group. Based on the computed binding energies and electronic spectra we reinterpret the experimental results in terms of four stationary state model, revealing the binding energies consistent with the theoretically computed ones. This new picture including fours states is more complicated than the initially assumed twostate one. Surprisingly, the nature of the excited states is dramatically different from the hydrogenic (s-, p-, d-) states known for the aqueous electron and previously assumed also for methanol. Charge-transfer states are responsible for the main absorption bands of the solvated electron in methanol, which explains the intensive absorption beyond $3.0 \mathrm{eV}$ and gives insights into the mechanism of excited state decay. These transitions can be rationalized with another simple model: assymetric double square-well.

\section{Acknowledgement}

This work is funded by the Swiss National Science Foundation (SNSF) Sinergia grant and the University Research Priority Program (URPP) for solar light to chemical energy conversion (LightChEC) of the University of Zurich. Computing resources were provided by the grant from Partnership for Advanced Computing in Europe under Project ID pr92 and a grant from the Swiss National Supercomputing Centre (CSCS) under Project ID uzh1. VRR has 
been supported by the SNSF in the form of Ambizione grant No. PZ00P2_174227. We thank Professor Jürg Hutter and Dr. Anna Hehn (University of Zurich) for the support and discussions.

\section{Supporting Information Available}

Details of the hybrid-functional calculations, MP2 calculations, electronic spectra, embedded calculations, volumetric data analysis and vertical electron binding energies are given in Supporting Information.

\section{References}

(1) Hart, E. J.; Boag, J. W. Absorption Spectrum of the Hydrated Electron in Water and in Aqueous Solutions. Journal of the American Chemical Society 1962, 84, 4090-4095.

(2) Herbert, J. M. Structure of the aqueous electron. Physical Chemistry Chemical Physics 2019, 21, 20538-20565.

(3) Lan, J.; Kapil, V.; Gasparotto, P.; Ceriotti, M.; Iannuzzi, M.; Rybkin, V. V. Simulating the ghost: quantum dynamics of the solvated electron. Nature Communications 2021, 12, 766 .

(4) Wilhelm, J.; VandeVondele, J.; Rybkin, V. V. Dynamics of the Bulk Hydrated Electron from Many-Body Wave-Function Theory. Angewandte Chemie International Edition 2019, 58, 3890-3893.

(5) Buttersack, T.; Mason, P. E.; McMullen, R. S.; Schewe, H. C.; Martinek, T.; Brezina, K.; Crhan, M.; Gomez, A.; Hein, D.; Wartner, G., et al. Photoelectron spectra of 
alkali metal-ammonia microjets: From blue electrolyte to bronze metal. Science 2020, 368, 1086-1091.

(6) Pizzochero, M.; Ambrosio, F.; Pasquarello, A. Picture of Wet Electron: A Localized Transient State in Liquid Water. Chemical Science 2019,

(7) Hara, A.; Yamamoto, Y.-i.; Suzuki, T. Solvated electron formation from the conduction band of liquid methanol: Transformation from a shallow to deep trap state. The Journal of Chemical Physics 2019, 151, 114503.

(8) Blandamer, M. J.; Shields, L.; Symons, M. C. R. 198. Unstable intermediates. Part XXVIII. Solvated electrons: rigid organic solvents. J. Chem. Soc. 1965, 1127-1131.

(9) Smith, D. R.; Pieroni, J. J. Detection of trapped electrons in organic glasses after gamma irradiation at $4.2 \mathrm{~K}$ by electron spin resonance spectroscopy. Canadian Journal of Chemistry 1967, 45, 2723-2727.

(10) Kumar, A.; Walker, J. A.; Bartels, D. M.; Sevilla, M. D. A Simple ab Initio Model for the Hydrated Electron That Matches Experiment. The Journal of Physical Chemistry A 2015, 119, 9148-9159, PMID: 26275103.

(11) Mones, L.; Turi, L. A new electron-methanol molecule pseudopotential and its application for the solvated electron in methanol. The Journal of Chemical Physics 2010, 132, 154507.

(12) Bartczak, W. M.; Kroh, J.; Sopek, M. Solvated electron in liquid methanol. An example of a statistical species in chemistry. Radiation Physics and Chemistry 1995, 45, 961970.

(13) Bartczak, W.; Sopek, M. Electron localization in liquid methanol. Quantum PathIntegral simulation. Polish Journal of Chemistry 1998, Vol. 72, nr 7S, 1798-1825. 
(14) Turi, L. A quantum chemical study of negatively charged methanol clusters. The Journal of Chemical Physics 1999, 110, 10364-10369.

(15) Mones, L.; Pohl, G.; Turi, L. Ab initio molecular dynamics study of solvated electrons in methanol clusters. Phys. Chem. Chem. Phys. 2018, 20, 28741-28750.

(16) Jou, F.-Y.; Freeman, G. R. Band resolution of optical spectra of solvated electrons in water, alcohols, and tetrahydrofuran. Canadian Journal of Chemistry 1979, 5\%, $591-597$.

(17) Jou, F.-Y.; Freeman, G. R. Shapes of optical spectra of solvated electrons. Effect of pressure. The Journal of Physical Chemistry 1977, 81, 909-915.

(18) Karashima, S.; Yamamoto, Y.-i.; Suzuki, T. Ultrafast Internal Conversion and Solvation of Electrons in Water, Methanol, and Ethanol. The Journal of Physical Chemistry Letters 2019, 10, 4499-4504, PMID: 31343891.

(19) Jacobson, L. D.; Herbert, J. M. Polarization-Bound Quasi-Continuum States Are Responsible for the "Blue Tail" in the Optical Absorption Spectrum of the Aqueous Electron. Journal of the American Chemical Society 2010, 132, 10000-10002, PMID: 20608656.

(20) Mosyak, A. A.; Prezhdo, O. V.; Rossky, P. J. Solvation dynamics of an excess electron in methanol and water. The Journal of Chemical Physics 1998, 109, 6390-6395.

(21) Walhout, P. K.; Alfano, J. C.; Kimura, Y.; Silva, C.; Reid, P. J.; Barbara, P. F. Direct pump/probe spectroscopy of the near-IR band of the solvated electron in alcohols. Chemical Physics Letters 1995, 232, 135-140.

(22) Shi, X.; Long, F. H.; Lu, H.; Eisenthal, K. B. Electron Solvation in Neat Alcohols. The Journal of Physical Chemistry 1995, 99, 6917-6922. 
(23) Sabatini, R.; Gorni, T.; de Gironcoli, S. Nonlocal van der Waals density functional made simple and efficient. Phys. Rev. B 2013, 87, 041108.

(24) VandeVondele, J.; Hutter, J. Gaussian basis sets for accurate calculations on molecular systems in gas and condensed phases. The Journal of Chemical Physics 2007, 127, 114105.

(25) Rybkin, V. V. Mechanism of Aqueous Carbon Dioxide Reduction by the Solvated Electron. The Journal of Physical Chemistry B 2020, 124, 10435-10441, PMID: 33170009.

(26) Rybkin, V. V.; VandeVondele, J. Spin-unrestricted second-order Møller-Plesset (MP2) forces for the condensed phase: from molecular radicals to f-centers in solids. Journal of Chemical Theory and Computation 2016, 12, 2214-2223.

(27) Del Ben, M.; Hutter, J.; VandeVondele, J. Forces and stress in second order MøllerPlesset perturbation theory for condensed phase systems within the resolution-ofidentity Gaussian and plane waves approach. The Journal of Chemical Physics 2015, $143,102803$.

(28) Del Ben, M.; Hutter, J.; VandeVondele, J. Electron Correlation in the Condensed Phase from a Resolution of Identity Approach Based on the Gaussian and Plane Waves Scheme. Journal of Chemical Theory and Computation 2013, 9, 2654-2671.

(29) Rybkin, V. V. Sampling Potential Energy Surfaces in the Condensed Phase with ManyBody Electronic Structure Methods. Chemistry, A European Journal 26, 362-368.

(30) Kühne, T. D. et al. CP2K: An electronic structure and molecular dynamics software package - Quickstep: Efficient and accurate electronic structure calculations. The Journal of Chemical Physics 2020, 152, 194103.

(31) Goedecker, S.; Teter, M.; Hutter, J. Separable dual-space Gaussian pseudopotentials. Physical Review B 1996, 54, 1703. 
(32) Ambrosio, F.; Miceli, G.; Pasquarello, A. Electronic levels of excess electrons in liquid water. The Journal of Physical Chemistry Letters 2017, 8, 2055-2059.

(33) Gaiduk, A. P.; Pham, T. A.; Govoni, M.; Paesani, F.; Galli, G. Electron affinity of liquid water. Nature Communications 2018, 9, 247. 


\section{Graphical TOC Entry}

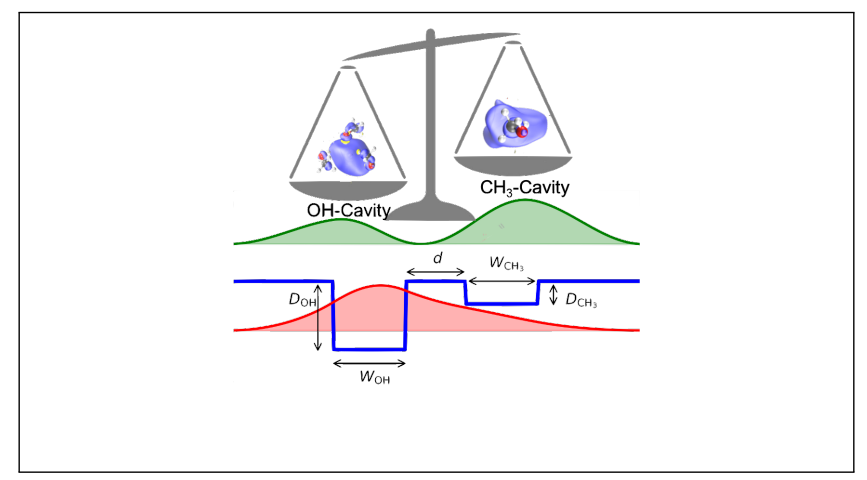

